\title{
全沙河工模型试验的研究
}

\author{
实 国 仡 \\ (永利审力帮 南京水利科学研究所)
}

在许多河道中, 常常不仅有悬沙输移, 也有底沙包括卵石推移, 在某些情况下还有异重流 形成. 在模型中单独复演某一种泥沙, 不可能很好解决复杂条件下的泥沙问题. 因此在研究 重大水利工程中的泥沙问题时, 需要在一个模型上同时复演各种形式的泥沙运动, 即进行悬沙 和底沙的综合试验。这种全沙河工模型试验, 国内以前没有进行过, 国外也没有成熟经验可供 参考. 我们于 1973 年对全沙模型相似律进行了探讨, 并于 1974 年完成了验证试验. 五年多 来的模型试验表明, 这种全沙模型在研究枢纽布置、电厂防沙、减少航道淤积和改善峡谷段航 行条件方面,是一个有效的工具.

在总结前人经验基础上，特别是依据作者本人近十几年来在泥沙运动理论方面所获得的 一些成果和进行正态及变态泥沙模型试验所取得的一些经验, 导得以下一些相似判据.

水流条件的相似在研究长河段泥沙淤积问题时, 首先必须保证水流在平面上的相似, 即垂线平均流速沿程和沿河宽分布的相似, 主流和回流的相似. 为此, 不论正态模型和变态模 型均需同时满足重力相似和阻力相似, 即

$$
\begin{aligned}
& \lambda_{\nu}=\lambda_{H}^{1 / 2}, \\
& \lambda_{c_{n}}=\left(\lambda_{l} / \lambda_{H}\right)^{2 / 2},
\end{aligned}
$$

式中 $\lambda_{v}$ 为流速比尺, $\lambda_{H}$ 为水深比尺, $\lambda_{l}$ 为平面长度比尺, $\lambda_{c_{n}}$ 为无尺度谢才系数比尺.

然而应当指出,不是在任何阻力形态的模型中都能真正同时满足重力相似和阻力相似的. 如果原型水流处于阻力平方区, 模型中的水流也必须处于阻力平方区或至少不在光滑区. 因 此要求模型中 $v_{*} \Delta / v \geqslant k$, 其中 $v_{*}$ 为摩阻流速, $\Delta$ 为粘率, $v$ 为液体粘滞系数. 当 $k \geqslant 60$ 时, 模型处于阻力平方区; 当 $k>3$ 时, 模型至少不在光滑区. 由此可得最小水深比尺如下

$$
\lambda_{H} \leqslant\left(\frac{v_{p} \Delta_{p}}{k c_{0 p} \nu_{m}}\right)^{1 / 5} \lambda_{l}^{7 / 10}
$$

对于正态模型, 则因 $\lambda_{H}=\lambda_{l}$ 而有

$$
\lambda_{l} \leqslant\left(\frac{v_{p} \Delta_{p}}{k c_{0 p} v_{m}}\right)^{2 / 3},
$$

式中角标 $p$ 表示原型量, $m$ 表示模型量.

虽然严格说来, 模型变态将引起流速沿垂线分布等以及垂直水流的偏离, 但只要变率不 大, 偏离是很小的,一般在测量误差范围之内. 关于流速沿垂线分布等问题,已为人们所熟知, 此处不赘述. 关于垂直水流问题也不难论证, 只要压强沿水深分布接近静水压强分布规律, 变 态模型中的垂直水流, 就与原型基本相似. 只是此时的垂直水流比尺 $\lambda_{v_{z}}=\lambda_{v} \lambda_{H} / \lambda_{l}$ 。 根据作

本文 1978 年 11 月 18 日收到。

第 14 期

科学 通 报 
者的研究,最大允许变率可由下式确定

$$
\lambda_{l} / \lambda_{H} \leqslant\left(1+B_{p} / 20 H_{p}\right),
$$

式中 $B_{p}$ 和 $H_{p}$ 分别表示原型的河宽和平均水深.

底沙运动相似根据能量原理, 作者导出了包括在床面推移和临底半悬移的底沙单宽 流量公式如下[1]

$$
q_{s b}=\frac{0.1}{c_{0}^{2}} \frac{\gamma \gamma_{s}}{\gamma_{s}-\gamma}\left(v-v_{k}\right)-\frac{v^{3}}{g c o},
$$

式中 $\boldsymbol{\gamma}$ 和 $\gamma_{s}$ 分别为水和沙粒的容重, $v$ 为平均流速, $v_{k}$ 为临界起动流速, $g$ 为重力加速度, $\omega$ 为沙粒沉速. 沙粒处于将动未动状态的临界起动流速可由下式确定 ${ }^{[1]}$

$$
v_{k}=0.265\left(\ln 11 \frac{H}{\Delta}\right) \sqrt{\frac{\gamma_{s}-\gamma}{\gamma} g d+0.19 \frac{\varepsilon_{k}+g H \delta}{d}},
$$

式中 $H$ 为水深, $d$ 为粒径, $\varepsilon_{k}$ 为粘结力参数, 对于天然沙 $\varepsilon_{k}=2.56$ 厘米 ${ }^{3} /$ 秒 $^{2}$, 对于无粘性颗 粒 $\varepsilon_{k}=0, \delta$ 为薄膜水厚度,其值为 $0.21 \times 10^{-4}$ 厘米, $\Delta$ 为床面䊁率, 对于平整河床, 当 $d \leqslant 0.5$ 毫米时, $\Delta=0.5$ 毫米, 当 $d>0.5$ 裹米时, $\Delta=d$, 对于不均匀沙用 $d_{30}$ 表示. 由底沙引起的 河床变形方程式为

$$
\frac{\partial q_{s b}}{\partial x}=-\gamma_{0} \frac{\partial Z}{\partial t}
$$

式中 $\gamma_{0}$ 为泥沙的干容重, $x$ 为沿程坐标, $z$ 为床面高程, $t$ 为变形时间. 由此得底沙运动的相 似条件为

和底沙冲淤时间比尺

$$
\begin{aligned}
\lambda_{q_{s b}} & =\frac{\lambda_{\gamma_{s}} \lambda_{v}}{\lambda_{r_{s-r}} \lambda_{c_{0}}^{2} \lambda_{\omega}}, \\
\lambda_{v} & =\lambda_{\nu_{k}},
\end{aligned}
$$

$$
\lambda_{t_{t}}=\left(\lambda_{r_{0}} \lambda_{H} \lambda_{l} / \lambda_{q_{s b}}\right),
$$

由于沙质底沙有可能处于半悬浮状态, 还应满足落淤部位相似(详见下文):

$$
\lambda_{\omega}=\lambda_{v} \lambda_{H} / \lambda_{l}
$$

悬沙运动相似 由于悬移质泥沙在运动过程中有超饱和及不饱和问题, 它的挟沙能力 与真实输沙量之间往往有很大差别. 因此在模拟悬沙运动时, 单纯模拟挟沙能力是不够的. 根据我们的研究 ${ }^{[2]}$, 恒定流的悬沙输移方程式具有如下形式:

$$
\begin{aligned}
& \frac{\partial(q S)}{\partial x}=\alpha \omega S_{*} B-\alpha \omega S B, \\
& \frac{\partial(q S)}{\partial x}=-\gamma_{0} B \frac{\partial z}{\partial t},
\end{aligned}
$$

式中 $q$ 为流量, $S$ 为用重量比表示的含沙量, $\alpha$ 为沉降机率, $S_{*}$ 为挟沙能力, $B$ 为河宽. 由上 述二式可得比尺关系如下:

$$
\begin{aligned}
& \lambda_{s}=\lambda_{s_{*}}, \\
& \lambda_{\omega}=\lambda_{\nu} \lambda_{H} / \lambda_{\alpha} \lambda_{l} .
\end{aligned}
$$

前者称为挟沙能力相似, 后者可称为沉降相似. 在适当选择模型沙时, 较易达到原型与模型中 的沉降机率值接近, 故 $\lambda_{\alpha} \approx 1$, 从而使 (15) 式与 (11) 式相同. 为了使悬沙相似还应尽量满 足扬动相似，即 


$$
\lambda_{v f}=\lambda_{v},
$$

扬动流速 $v_{f}$ 可由下述二式之一确定, 采用其中数值较大的一个:

或

$$
\begin{aligned}
& \lambda_{v f}=1.5\left(\ln 11 \frac{H}{\Delta}\right) \sqrt{\frac{\gamma_{s}-\gamma}{\gamma} g d} \\
& \lambda_{v_{f}}=0.408\left(\ln 11 \frac{H}{\Delta}\right) \sqrt{\frac{\gamma_{s}-\gamma}{\gamma} g d+0.19 \frac{\varepsilon_{k}}{\gamma}+g H \delta}
\end{aligned},,
$$

由 (13) 式得悬沙的冲淤时间比尺 $\lambda_{t_{2}}$ 为:

$$
\lambda_{t_{2}}=\lambda_{r_{0}} \lambda_{l} / \lambda_{v} \lambda_{s}
$$

我们根据维立干诺夫 ${ }^{[3]}$ 和巴连布拉特 ${ }^{[4]}$ 的悬沙理论, 得到了确定悬沙挟沙能力的比尺:

$$
\lambda_{s_{*}}=\frac{\lambda_{r_{s}}}{\lambda_{r_{s-r}}} \frac{\lambda_{i} \lambda_{v}}{\lambda_{\omega}}
$$

式中 $i$ 为水面比降. 当同时满足重力相似、阻力相似和沉降相似时, 上式为

$$
\lambda_{s_{*}}=\lambda_{r_{s}} / \lambda_{r_{s-r}}
$$

异重流相似 在含有细颗粒泥沙的河流，在一定条件下，在一定区域内能够形成异重 流,因此全沙模型也要满足异重流相似条件. 异重流的发生条件为

异重流的输沙量变化及淤积变化分别为

$$
\frac{v^{2}}{\frac{\gamma^{\prime}-\gamma}{r^{\prime}} g H}=k_{1},
$$

$$
\begin{aligned}
& \frac{\partial\left(v_{e} S_{e} H_{e}\right)}{\partial x}=\omega S_{\epsilon}, \\
& \frac{\partial\left(v_{e} S_{\varepsilon} H_{e}\right)}{\partial x}=-\gamma_{0} \frac{\partial z}{\partial t},
\end{aligned}
$$

在上述二式中 $\gamma^{\prime}$ 为浑水容重, 角标 $e$ 表示异重流各量. 由于

$$
r^{\prime}=\gamma+\left(\frac{r_{s}-\gamma}{\gamma_{s}}\right) s,
$$

从而可得异重流的发生条件相似判据为

$$
\lambda_{H} \frac{\lambda_{r_{s-r}}}{\lambda_{r_{s}}} \lambda_{s}=\lambda_{v 。}^{2}
$$

即当满足重力相似时与悬沙相似条件 (20) 式相同. 此条件实际上是要求模型浑水的容重与 原型浑水容重相同, 这也是达到异重流相似最关键的一个条件. 由 (21) 式得到的异重流淤积 部位相似条件与 (11) 式相同. 异重流淤积的时间比尺由 (22) 式及 (24) 式得

$$
\lambda_{t_{3}}=\frac{\lambda_{r_{0}} \lambda_{l} \lambda_{r_{s}}^{3 / 2}}{\lambda_{s}^{3 / 2} \lambda_{r_{s-s}}^{1 / 2} \lambda_{H}^{1 / 2}}
$$

悬沙与底沙的统一问题 悬沙与底沙, 包括异重流和卵石在内, 能否在一个模型中复 演, 关键在于各种泥沙的冲淤时间比尺能否一致。将 (8) 式代人 (10) 式并在满足重力相似、 阻力相似和冲淤部位相似时, 则底沙的冲淤时间比尺可以写作

$$
\lambda_{t_{1}}=\lambda_{r_{0}} \lambda_{r_{s-r}} \lambda_{l} / \lambda_{r_{s}} \lambda_{v_{0}}
$$

当悬沙的含沙量比尺用 (20) 式确定时, 则悬沙的冲淤时间比尺 $\lambda_{t}$, 具有与上式完全相同的形 式. 当同时满足重力相似、阻力相似和浑水容重相同时, 异重流的淤积时间比尺 $\lambda_{t_{3}}$ 也具有与 
（26）式完全相同的形式. 如果采用同一种模型沙模拟悬沙和底沙时，则悬沙、底沙和异重流 的干容重比尺 $\lambda_{r_{0}}$ 就有可能完全相同或相近，因而三种泥沙的冲淤时间比尺就完全或基本相 同. 由此可见,只要同时满足上述各相似条件, 就可以在一个模型中同时复演全部泥沙及其冲 淤情况。

利用上述相似条件，我们为长江葛洲坝水利枢纽设计了一个长河段的全沙模型. 用电木 粉做模型沙, 其容重为 1.4 吨/米 ${ }^{3}$, 其粒径级配可根据要求制作. 根据电木粉的起动、沉降和阻 力特性, 决定选用平面比尺为 1:200 垂直比尺为 1:100 的变态模型. 按前述相似条件计算, 这个模型基本上满足了重力相似、阻力相似、悬沙和底沙的沉降相似、含沙量和输沙量相似、 异重流的发生部位相似和淤积相似、悬沙的扬动相似、底沙和卵石的起动相似等. 各种泥沙 的冲淤时间比尺约为 98 . 为了操作上的方便, 我们采用冲淤时间比尺为 96 , 即模型中的 15 分钟相当于原型的一天.

为了减少模型中的加沙数量以便于进行多年长系列淤积试验，模型中的悬沙采用了㡒环 系统,但推移质泥沙和卵石则采用模型进口加沙尾部沉沙的方式. 对于这种全沙试验, 关键问 题是确保模型进口处的泥沙级配与要求一致。通过实践我们解决了这个问题，使进入模型的 各种泥沙基本上与要求级配一致(见图 1).

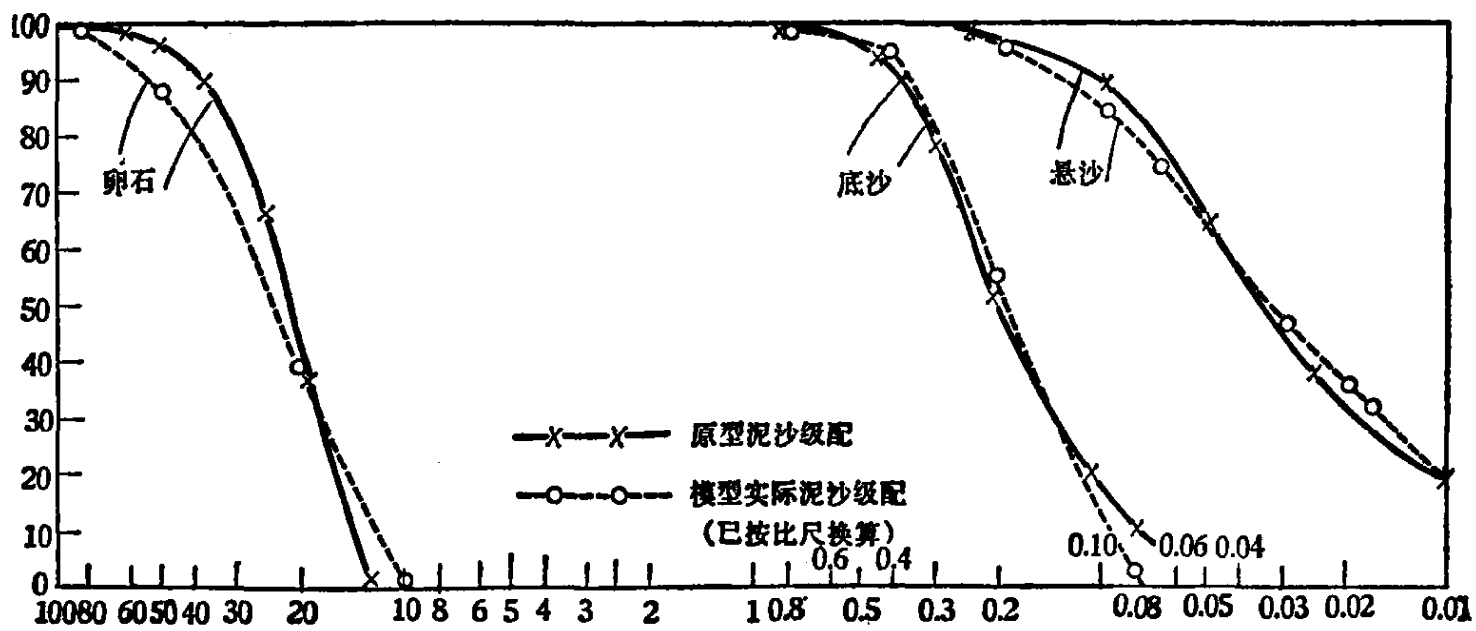

图 1 模型中各种泥沙与原型比较(毫米)

验证试验表明, 按前述相似理论设计模型, 是可行的. 模型上挟带全沙的水流情况, 包括 水位、流速分布、回流范围和泡漩高度，与原型基本相同. 含沙量分布、底沙和卵石的运动规 律，冲淤部位和淤积数量与原型基本一致(见图 2). 异重流的形成部位和淤积分布也与原型

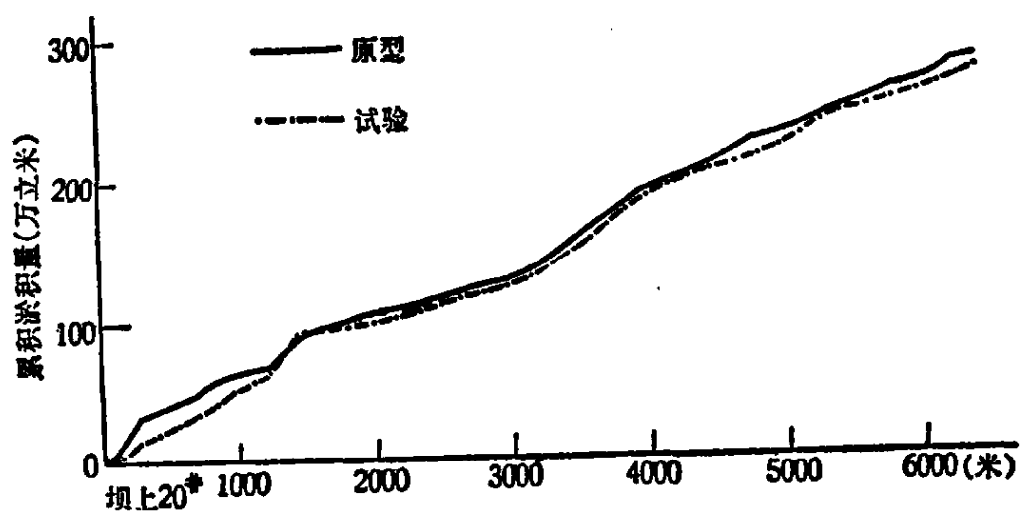

图 2 沿程䒺积淤积量比较 


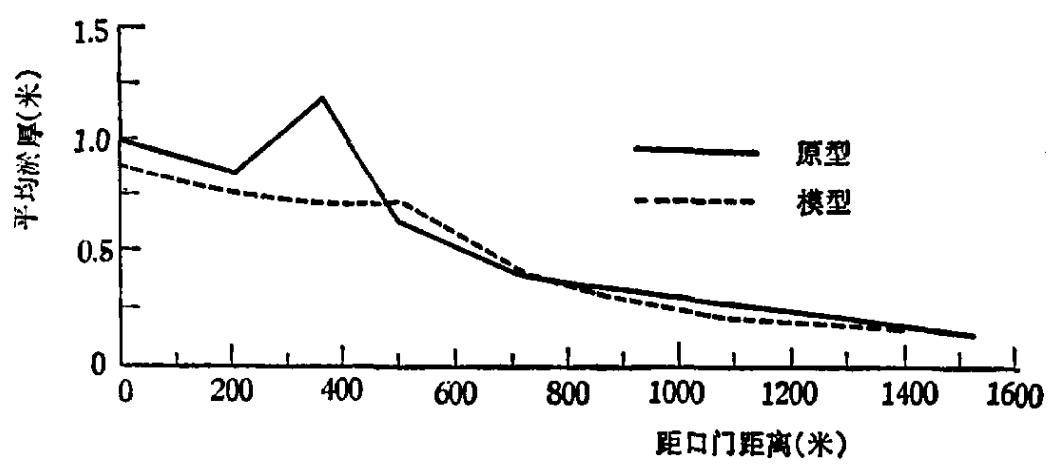

图 3 三江异重流平均淤积厚度沿程分布

一致 (见图 3)。各淤积区中的淤沙级配与原型淤沙级配也很相近(见表 1)。这说明模型中悬 沙和底沙的淤积比例与原型基本一致.

表 1

\begin{tabular}{|c|c|c|c|c|c|c|c|c|c|c|c|}
\hline \multicolumn{2}{|c|}{ 取样位 置 } & 坝上 17 & 坝上 12 & 坝上 5 & 水文䉼面 & 上围㘿 & 二江口 & 三江 10 & 三江 9 & 三江 8 & 三江 7 \\
\hline \multirow{2}{*}{ 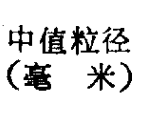 } & 原 型 & 0.282 & 0.187 & 0.205 & 0.190 & 0.031 & 0.036 & 0.030 & 0.029 & 0.027 & 0.018 \\
\hline & 模 型 & 0.264 & 0.202 & 0.199 & 0.154 & 0.032 & 0.035 & 0.041 & 0.037 & 0.036 & 0.031 \\
\hline
\end{tabular}

\title{
参考文献
}

[1] Доу Го-жень (窦国仁), K теории трогания частиц наносов, 中国科学, 11 (1962),7,

[ 2 ] 窨国仁, 潮汐水流中的悬沙运动及冲淤计算, 水利学报, 1963,4 .

[ 3 ] Великанов, М. А., Динамика русловых потоков, II (1955).

[4] БаренЄлатт, Г. И., О движении взвешенных частиц в турбулентном потоке, Прикладная метематика и механика 17 (1953).

\section{AN INVESTIGATION ON THE TOTAL SEDIMENT TRANSPORT MODEL OF SANDY RIVER}

\author{
Dou GuO-REN（窦国仁）
}

\begin{abstract}
This paper presents the similarity of eriteria for carrying out simultaneously bed load, suspended load and density current experiments in a single model of sandy river. Some results regarding the sediment movement theory available have been used in studying the similarity of conditions for various forms of sediment transport. The experimental data indicate that the model designed on this theory similarity is similar in the main to the propotype with respect to current, suspended load, bed-load movement, density current, location and quantity of scouring and deposition, and grain size of sand in river channels.
\end{abstract}

